DOI: $10.30972 /$ eitt.704765

\title{
Contaminación lumínica: la iluminación Led. Un análisis del conocimiento actual de sus efectos sobre plantas y animales
}

\author{
José L. Fontana, E. F. Scozzina, V.Marder, J.L.Ramírez \& A. de J. Lin \\ Facultad de Ciencias Exactas y Naturales y Agrimensura, UNNE.
}

\section{Resumen}

La contaminación lumínica tiene su origen reciente con la invención de la lámpara incandescente y se ha intensificado enormemente con el crecimiento de las poblaciones y el incremento de la iluminación con distintas tecnologías hasta llegar al desarrollo de la tecnología led con utilización de la luz blanca, cuyo componente principal es la longitud de onda correspondiente al azul. Desde entonces los estudios sobre sus efectos sobre el medio y sobre os organismos fueron en aumento, demostrando las consecuencias positivas y negativas sobre el medio y los organismos. Las ventajas de las tecnologías de iluminación por diodos emisores de luz o leds, tienen que ver con la economía energética, la duración de las lámparas, la intensidad y la posibilidad de variar los colores, aspectos que permiten una gran amplitud de aplicaciones domésticas, urbanas e industriales. Son reconocidas los efectos negativos con respecto a sus incidencias en el reloj biológico, en la reproducción, en la dinámica de las poblaciones, $\mathrm{y}$ en la salud de animales, incluyendo el hombre, que llevan a pensar en soluciones para minimizarlos.

En este trabajo analizamos los conocimientos actuales de los efectos de estas tecnologías de iluminacion sobre el medio y sobre animales y plantas.

Palabras clave: led, efectos, animales, plantas, conocimiento actual. 


\section{Summary}

Light pollution has its recent origin with the invention of the incandescent lamp and has intensified enormously with the growth of populations and the increase in lighting with different technologies, leading to the development of LED technology using white light whose component main is the wavelength corresponding to blue. Since then studies on its effects on the environment and on organisms have been increasing, showing the positive and negative consequences on the environment and organisms. The advantages of lighting technologies by light-emitting diodes or LEDs, have to do with energy economy, lamp life, intensity and the possibility of varying colors, aspects that allow a wide range of domestic, urban and industrial applications. The negative effects regarding their effects on the biological clock, on reproduction, on population dynamics, and on the health of animals, including man, are recognized, which lead to thinking of solutions to minimize them.

In this work we analyze the current knowledge of the effects of these lighting technologies on the environment and on animals and plants.

Keywords: led, effects, animals, plants, current knowledge.

\section{Introducción}

El diodo emisor de luz o led (light-emitting diode) parece haberse transformado los últimos años en la panacea para un buen aprovechamiento de la energía. Muy eficientes comparados con otras fuentes de luz, pueden emitir cualquier tipo de color; son una fuente de luz fría, ya que muy poco de la energía se transforma en calor, y tienen una larga vida útil. Todas estas ventajas llevaron a incentivar su uso tanto en la iluminación doméstica, como urbana, industrial y producción de alimentos. La revolución del led, quedó plasmada en el Premio Nobel de Física a los japoneses I.Akasaki, H.Amano y S.Nakamura en 2014 por inventar el led azul en la década de 1990, que permite una luz muy brillante, eficiente, desde el punto de vista energético, y por lo tanto considerada "amigable con el ambiente". Pero este ahorro de energía no está excento de efectos directos y secundarios sobre los organismos, tanto plantas como animales. Los últimos años se han publicado numerosos estudios que demuestran los efectos nocivos de la iluminación por Leds, que han llevado a distintas instituciones de tratar de establecer nomas de fabricación y de uso. Así los 
leds de luz blanca, por el elevado porcentaje de luz azul que emiten, son los menos recomendables por los motivos que enumeraremos más adelante (ver resultados), en base a estudios realizados en su mayoría por europeos y norteamericanos. En Argentina estos estudios son muy raros.

La luz artificial, y particularmente la led, tiene numerosos efectos sobre plantas y animales, efectos positivos como su utilización en la producción vegetal, o negativos como las modificaciones del reloj biológico de muchas especies, afectando la actividad. La secuencia natural de noche y día fue alterada profundamente en poco tiempo, si se considera el punto de vista evolutivo de los organismos, por la iluminación artificial, en constante aumento. Kyba et al. (2017) establecieron que entre 2012 y 2016 las regiones iluminadas en el planeta crecieron 8,8\% en extensión y con un aumento en el brillo desde que apareció la tecnología Led de 1,8\%/año. Y Argentina se ubicó en el top ten a escala mundial, como país con mayor contaminación lumínica, tanto que el 57,7\% de los argentinos “no logra ver las estrellas a causa de cielos nocturnos extremadamente brillantes" ( www.perfil.com).

En este análisis resumiremos los principales resultados obtenidos hasta el presente y los estudios en marcha sobre la contaminación lumínica y los efectos sobre el ambiente, las plantas y animales, y el hombre. Haremos especial incapié en la tecnología Led, por su reciente y rápida difusión como sistema adoptado por todas las ciudades y promocionado desde los organismos estatales.

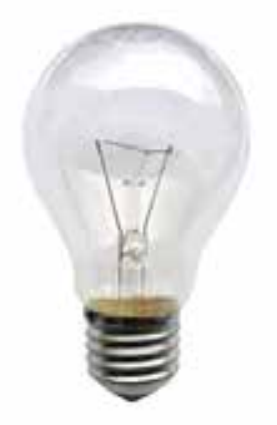

incandescente

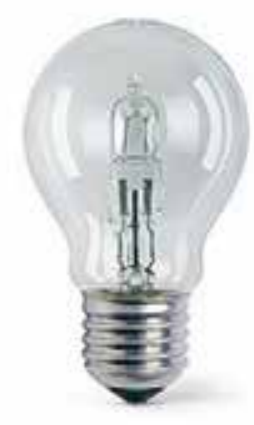

halógena

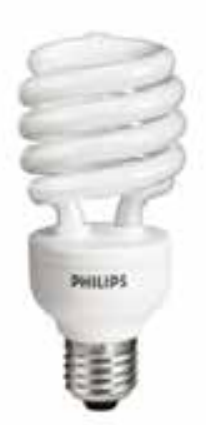

de bajo consumo

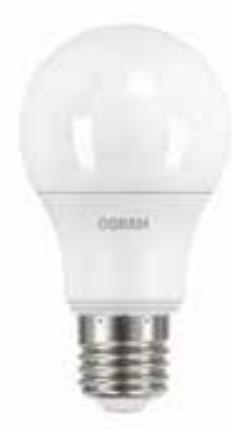

led

Figura 1. Distintos tipos de lámparas. 


\section{Métodos}

Se analizaron numerosas publicaciones de autores locales y extranjeros publicados hasta el presente referidos a la contaminación lumínica, particularmente la iluminación led, sus efectos y consecuencias sobre plantas y animales, incluyendo el hombre. Fueron consultadas páginas de organismos especializados y de instituciones públicas que se han ocupado del tema, así como aquellas que transcribieron entrevistas a especialistas.

\section{Resultados}

Mucho cambió la iluminación artificial desde que Edison presentó publicamente su invento, iluminando la Christie Street en Menlo Park, con sus bombillas incandescentes, la última noche de 1879. Desde entonces, la noche urbana nunca más fue oscura y ver las estrellas empezó a volverse cada vez más difícil. Un adelanto tecnológico enorme que fue evolucionando hasta desarrollar lámparas de sodio, halógenas, de bajo consumo y de led, tecnología que se volvió predominante. Fue aumentando la intensidad, menor generación de calor y disminuyó el consumo, aspectos a primera vista ambientalmente aceptables. Pero no tardaron en aparecer los problemas con consecuencias para la biodiversidad.

\section{Los Leds y la biodiversidad}

En el Convenio sobre Diversidad Biológica (1992) se la define como "la variabilidad de organismos vivos de cualquier fuente, incluidos, entre otros, los ecosistemas terrestres y marinos y otros sistemas acuáticos, y los complejos ecológicos de los que forman parte; comprende la diversidad dentro de cada especie, entre las especies $y$ de los ecosistemas". Es decir abarca animales, plantas, bacterias, hongos, los genes.

¿Los Leds influyen sobre la biodiversidad? Esta tecnología es a primera vista muy beneficiosa para el ambiente por el enorme ahorro de energía. Sin embargo, "no todo lo que brilla es oro". La contaminación lumínica es un efecto estudiado antes de la aparición de los leds, y con esta nueva tecnología, que implica luz de alta intensidad y un fuerte componente del azul, el problema se agudiza. Numerosos estudios de las consecuencias ambientales de su utilización doméstica y pública, dan a conocer problemas registrados con aves, insectos, arácnidos, con el hombre. 


\section{Aplicaciones generales de la tecnología LED}

- Semáforos: las lámparas Leds hacen bien visible la luz, aún en pleno día; son más duraderas que las lámparas incandescente, y energéticamente mas sustentables.

- Iluminación pública: para calles y edificios se utiliza por lo general el blanco (Fig.2).

- Iluminación domiciliaria: uso doméstico incentivado por intensidad y bajo consumo.

- Cartelería de publicidad fija: el uso permitió una excelente visibilidad diurna de estos carteles y la posibilidad de anuncios animados, con bajo consumo y mantenimiento.

- Pantallas de led para información: utilizadas en lugares públicos para dar información específica (horarios, salidas, llegadas en aeropuertos, instituciones).

- Luces de emergencia: específicamente utilizadas en ambulancias, móviles de la policia y de bomberos, vehículos de seguridad y de auxilio.

- Pantallas de computadora (monitores), celulares, indicadores de encendido/apagado de artefactos eléctricos.

- Luces de vehículos: de gran alcance y brillo.

- Luces de advertencia: instalaciones industriales, chimeneas, antenas, edificios...

- Usos militares.

- Iluminación para industrias: un gran ahorro energético y una muy buena iluminación.

- Iluminación para invernaderos, criaderos, etc.: uso en producción de alimentos; contribuye al ahorro energético, y posibilidad de modificar intensidad y espectro.

\section{Aplicaciones del led que involucran organismos}

A pesar del reciente desarrollo de la luz azul de tecnología Led, son numerosas las aplicaciones en desarrollo de plantas, horticultura, invernaderos, criaderos de aves, control de insectos, estimación de cosechas, etc. La iluminación por leds permite el manejo de su espectro para darle un fin específico, por ej., formación de hojas, floración, fructificación, crecimiento, calidad nutritiva (Malagamba Stiglich, 2015). 

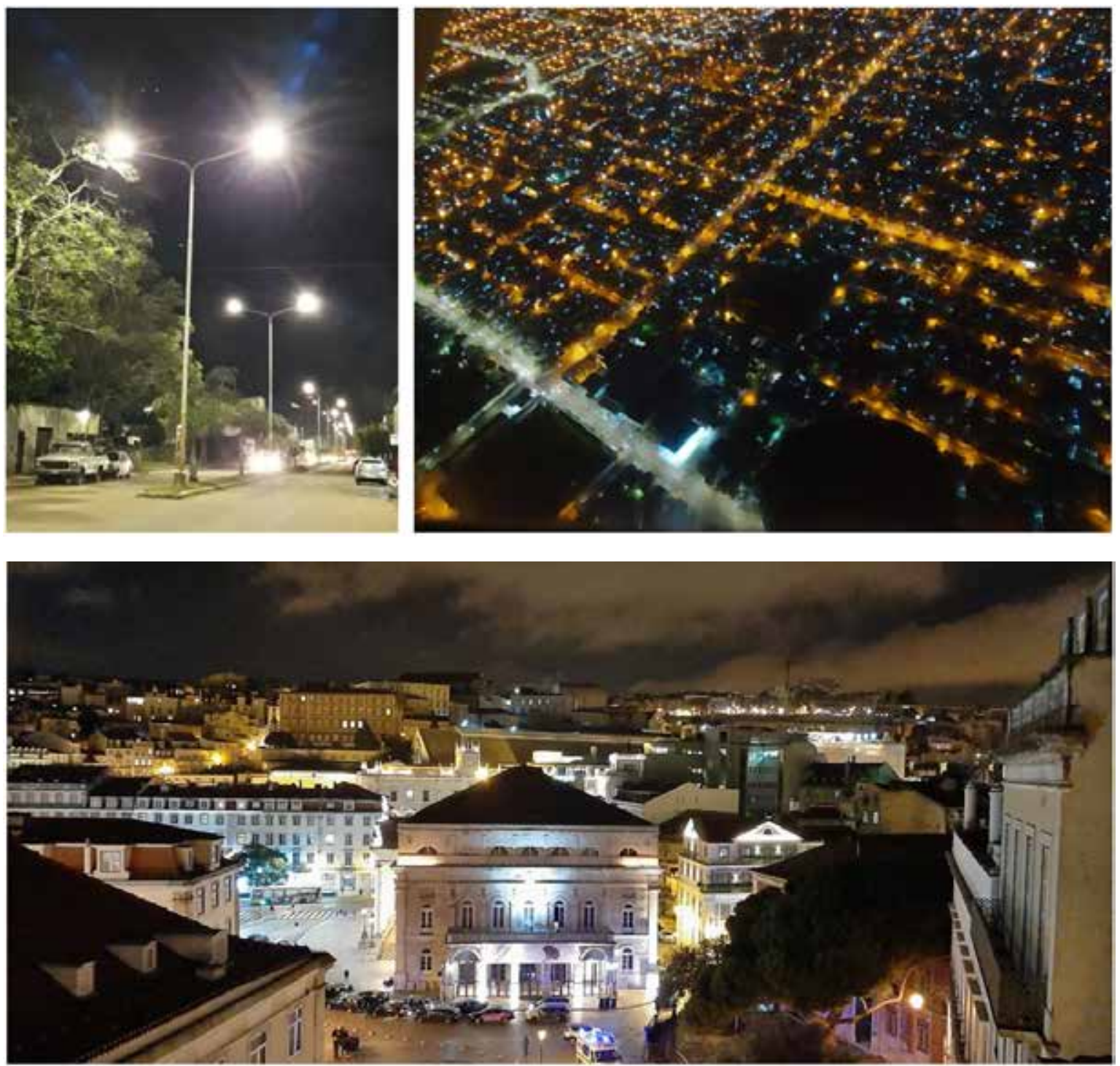

Figura 2. Contaminación lumínica. Arriba, calle iluminada con luminarias Leds (foto J.L. Fontana), y vista aérea nocturna (foto Escuela de Vuelo Corrientes), ambas de la ciudad de Corrientes. Abajo, la ciudad de Lisboa con iluminación ornamental de edificios (foto Nancy Fontana); obsérvese el resplandor de la luz en las nubes.

En horticultura. La iluminación Led en horticultura representó una revolución productiva (Alvarez, 2019), dada la capacidad de controlar el espectro, intensidad y dirección, junto a la enorme economía energética. Los ajustes del espectro permiten una eficiencia puntual en la planta, lo que redunda en una mayor producción y una mejora del valor nutricional de las plantas, y una mayor efectividad de la luz. La utilización específica de radiaciones del rojo y azul para los invernaderos con iluminación led es la responsable de la tonalidad púrpura de la luz observada en los sistemas de cultivo. Permite el manejo del ritmo de crecimiento modificando la 
duración de horas de luz, permitiendo su cultivo incluso durante invierno en invernadero. Esto fue experimentado con la planta comestible "bambú de agua" (Zizania latifolia) en Taiwán (Alvarez, 2020) mediante la iluminación Led evitando la caida de hojas y aumentando la producción.

En floricultura. En experimentos realizados (Pachacama Moreno, 2018) se demostró la importancia de la aplicación de la luz led blanca para la mayor formación de tallos florales en invernaderos dedicados a la floricultura.

Ayuda en agricultura. La empresa Osram lanzó un Led de infrarrojo cercano que puede ser incorporado a un teléfono celular o a una tablet. Mediante un escaneado del cultivo el productor puede planificar el momento exacto de cosecha. Brinda información sobre el contenido de azúcar, grasa y agua (https://smart-lighting.es/osram-lanza-nuevo-led-infrarrojo-cercano-ayudar-los-agricultores-planificar-mejor-momento-la-cosecha/).

Lámparas menos atractivas para insectos. El desarrollo de lámparas Led que atraen menos a los insectos, muchos de ellos transmisores de enfermedades. Estas lámparas utilizan una determinada longitud de onda que no resultan atractivas para estos animales (Cavanillas, 2015), contribuyendo por lo tanto a la salud pública.

\section{Beneficios}

Larga duración. Las lámparas de Led son publicitadas como artefactos de una vida útil muy larga, lo que supone ahorro de materiales y una huella de carbono menor. Superan entre 3 y 25 veces a cualquier otro tipo de lámparas (tabla 1). Con un promedio de uso de 8 hs/día, una lámpara led tendría una vida útil de unos 17 años, pero depende de los materiales de fabricación, forma de uso, lugar de instalación, tensión eléctrica constante, diseño del disipador de calor, etc., considerando además que las mediciones se realizan en condiciones controladas de laboratorio.

En realidad, es posible una duración mucho mayor de las bombillas incandescente como lo atestigua la que está encendida sin interrupción desde 1901 en el 
cuartel de bomberos de Livermore (California). La limitación de la vida útil fue un acuerdo de fabricantes a través del pacto extraoficial denominado "cartel Phoebus", firmado por Osram, Philips y GE en diciembre de 1924.

Tabla 1. Vida útil media de distintos tipos de lámparas.

Fuente: https://dmdiluminacion.com/vida-util-led-cuantos-anos-dura-una-lampara/

\begin{tabular}{|l|l|}
\hline Tipo de lámpara & horas de vida útil \\
\hline Lámparas incandescentes & $1000 / 2000$ \\
\hline Lámparas de descarga y fluorescentes & $4000 / 8000$ \\
\hline LED & $15.000 / 100.000$ \\
\hline
\end{tabular}

Bajo consumo. Ahorro considerable de energía (hasta un $80 \%$ en relación a lámparas incandescentes tradicionales). Hernández Moreno (2015) concluye que el consumo de una lámpara incandescente es de 5,55 veces más que la fluorescente y 8,10 veces más que una Led; con el ciclo completo (fabricación, uso y disposición final) una lámpara incandescente contribuye al calentamiento global 29,87 veces más que la fluorescente y 146,82 veces más que una Led, lo que reduce considerablemente la huella de carbono.

No contienen elementos peligrosos. Son menos contaminantes que otras lámparas. No continen mercurio como las fluorescentes por ejemplo, pero necesitan ser recicladas ya que contienen otros elementos electrónicos que también son contaminantes.

Reducción de las emisiones de CO2. Los Led son considerados ambientalmente más aceptables ya que en su fabricación se utiliza una cantidad menor de materiales, dado su pequeño tamaño, que unido a su mayor durabilidad y eficiencia, reduce la cantidad de residuos, y por ende, la de subproductos como el $\mathrm{CO} 2$.

\section{Desventajas. Efectos negativos}

\section{Sobre el ambiente.}

La contaminación lumínica de los cielos. Las ciudades emiten luz que se refleja en las partículas de la atmósfera, impidiendo una clara visión del cielo nocturno. Los observatorios astronómicos se emplazan en sitios libres de esta contaminación 
para permitir una visión perfecta. La International Dark Sky Association (IDA) tiene como objetivo proteger los cielos oscuros, y llevar adelante acciones de mitigación de la contaminación lumínica. Esta contaminación lumínica no sólo impide la investigación, sino también la recreación de la gente que disfruta de contemplar el cielo nocturno ("el derecho a mirar las estrellas").

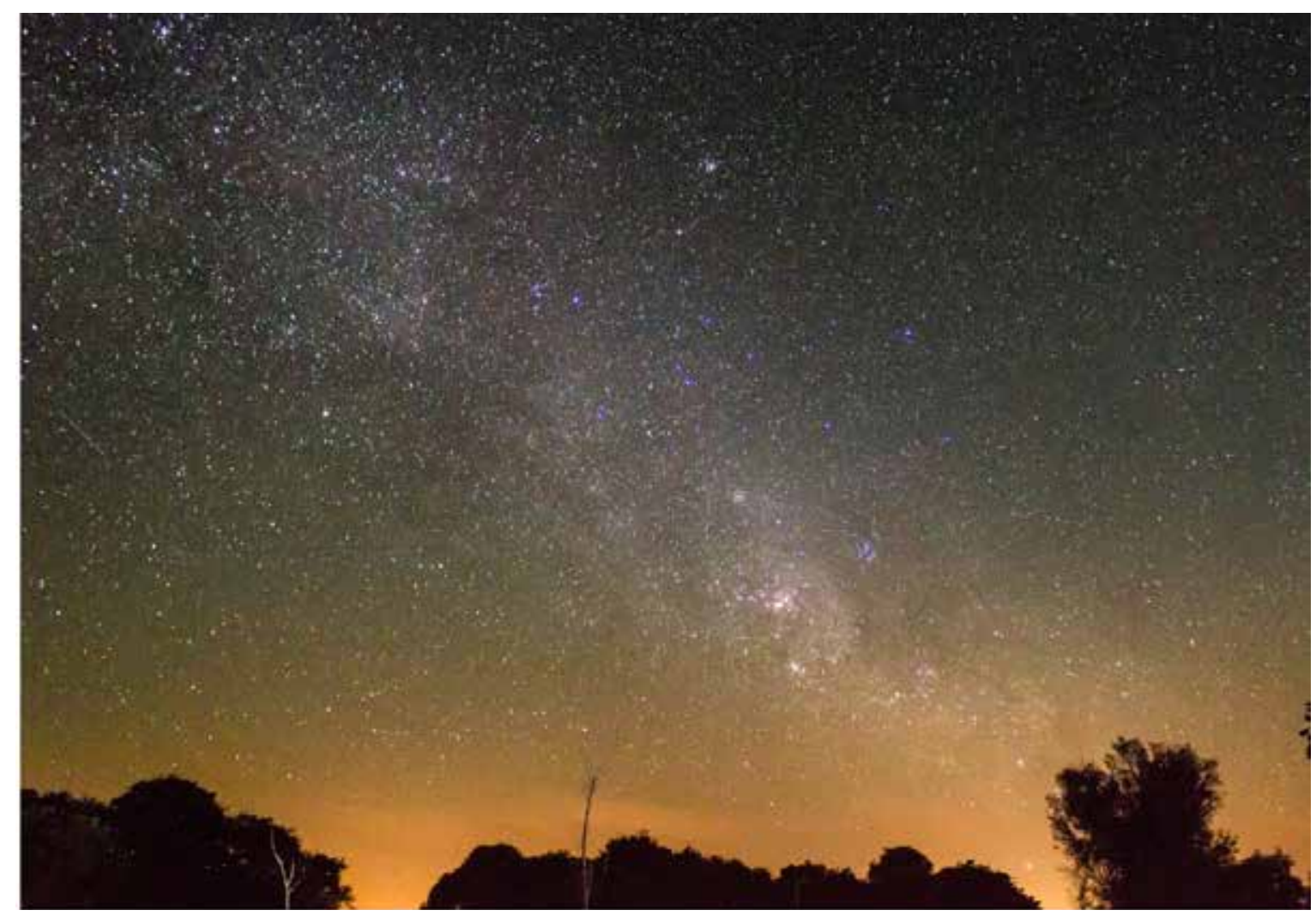

Figura 3. Cielo nocturno sin contamincación lumínica. Tartagal, norte de Santa Fe (foto Damián Feresín).

En 2007 en la Conferencia Internacional en Defensa de la Calidad del Cielo Nocturno y el Derecho a Observar las Estrellas (La Palma, Islas Canarias), apelaron a la comunidad internacional, gobiernos, instituciones públicas y privadas y al mundo de la ciencia y de la cultura, a adoptar un serie de principios y objetivos contenidos en la Declaración sobre la defensa del cielo nocturno y el derecho a la luz de las estrellas. Entre otros sugiere que "El control de la contaminación lumínica debe por lo tanto ser un requisito básico en las políticas de conservación de la naturaleza, incorporando esta dimensión en la gestión de las áreas protegidas, garantizando de forma más efectiva la protección del medio natural y la conservación de la diversidad biológica”. 
La contaminación lumínica de calles y hogares. Las pantallas Led de publicidad de fuerte intensidad han sido responsables de numerosos accidentes de tránsito, y pueden ser visibles desde varios cientos de metros, alterando además el paisaje urbano. Siguen activadas toda la noche, cuando su efecto publicitario es casi nulo, no así el consumo energético. Se convierte también en una "luz intrusa" en los hogares, alterando la tranquilidad y el sueño. Estos problemas reales condujeron a la elaboración de normas, como la municipalidad de Asunción que obliga a reducir la intensidad de las pantallas led de publicidad a 200 lúmenes entre las 18 y las 6 horas (ordenanza 121/2017).

\section{Sobre animales}

Sobre los tiempos biológicos. La iluminación nocturna que genera grandes espacios donde la noche prácticamente desaparece, y cuyo resplandor visible a muchos $\mathrm{km}$ de distancia, condujo a modificaciones de los ciclos naturales de las especies (Gaston et al., 2018). Estos ciclos, que implican una duración de horas de luz y oscuridad, variable a lo largo del año con las estaciones, han sufrido un cambio brusco por efecto de la iluminación en los últimos 100 años. Animales diurnos que ven afectados su ritmo de descanso, o animales de vida nocturna que quedan visibles y que naturalmente se amparaban en la oscuridad para escapar de sus depredadores.

Modificación del equilibrio natural de poblaciones animales. Thomas et al. (2017) estudiaron la dinámica de poblaciones de arácnidos y sus insectos presa en pastizales templados de Inglaterra bajo infuencia de iluminación led idéntica a las utilizadas en las ciudades, variando espectro de colores, intensidad y duración. Comprobaron la fuerte atracción que escarabajos y arañas depredadoras tienen por este tipo de iluminación, lo que incidiría en la reducción de otras poblaciones de insectos. Llegaron a la conclusión que para evitar impactos sobre los organismos, la única solución sería evitar el uso de Leds y cualquier tipo de otra iluminación, ya que reducir intensidad o apagarlas durante varias horas en la noche, no eliminaba totalmente su efecto negativo.

Efectos sobre las aves. La iluminación de calles con tecnología Led, aumenta la actividad nocturna de aves de hábitos diurnos. En una entrevista (Info Blanco sobre Negro, 2019), la Lic. en Biología L. Carettoni comentó que en la ciudad de La 
Plata, “... comprobé que a las dos de la mañana los zorzales y los chingolos están cantando. La verdad es que eso no es normal”, y responsabilizó al nuevo tipo de iluminación. Son conocidos los efectos sobre aves migratorias. Durante la noche se guían por la posición de las estrellas. Al estar menos visibles por la fuerte intensidad luminosa de las grandes ciudades, producen desorientación en los migrantes, lo que significa problemas para llegar a las zonas de alimentación y de reproducción.

Alteraciones en la reproducción. La luz artificial puede provocar la interrupción de los patrones de reproducción en los walibies australianos en libertad (Robert el al., 2015), reduciendo los niveles de melatonina, hormona reguladora del sueño y tal vez de la fisiología reproductora (Lissette Ricaurte Galindo, 2005).

En organimos costeros o marinos. Aronson (2020) describe efectos nocivos de la iluminación costera sobre ciclos de apareamiento en peces, particularmente aquellos que llegan a las playas para desovar, y las redes tróficas a lo largo de las costas del sur de California. Al chorlito nevado, un ave costera, le es difícil encontrar playas sin iluminación para la postura de huevos. Avenidas costeras iluminadas, playas con reflectores y negocios gastronómicos con carteles de luz intensa contribuyen con efectos negativos para las especies que necesita de un ambiente sin contaminación lumínica para completar su ciclo reproductivo. Por efecto de la iluminación, las crías de tortugas marinas se dirigen hacia el continente en lugar de hacerlo hacia el mar. La pulga de mar Talitrus saltamontes se guía por la luz del sol y de la luna para sus deplazamientos mar-playa; la iluminación artificial según su intensidad puede ser interpretada como sol o como luna (Ugolini et al., 2012). Se ha medido una mayor tasa de alimentación de aves zancudas en zonas costeras iluminadas, modificando su ritmo alimenticio normal (Santos et al., 2010). Los corales sincronizan el desove a ciclos de la luz de la luna (T.W.Davies: proyecto ALICE). La luz azul de la iluminación led penetra a mayor profundidad que la luz de otras tecnologías por lo que puede esperarse una mayor influencia sobre los organismos marinos.

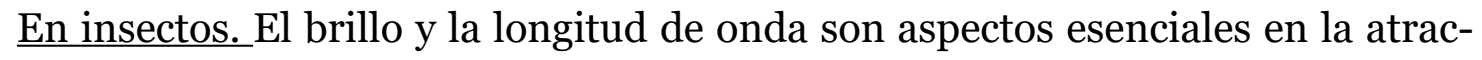
ción de los insectos; se sienten fuertemente atraídos por la luz artificial, particularmente por los Leds (Stokstad, 2017). Según Alvarez (2018), la mitad de las especies de insectos son nocturnas y dependen de la oscuridad, la luz de la luna y de las 
estrellas para orientarse y ocultarse de sus depredadores. La iluminación artificial cambia su medio y altera el comportamiento, quedando visibles a sus depredadores. Menos insectos significa menos polinizadores; la mayor y más fácil disponibilidad de alimento para depredadores puede determinar variaciones en poblaciones de insectos potencialmente dañinos.

Los insectos que buscan el agua para alimentarse o reproducirse se valen de la luz polarizada por lagunas, arroyos y otros cuerpos para encontrarla. De ahí la fuerte atracción por las luces de los automóviles (https://smart-lighting.es/4-formas-frenar-contaminacion-luminica-insectos/).

\section{Sobre plantas}

El fotoperíodo o alternancia entre luz y oscuridad a la que se somete diariamente la planta determina procesos que se realizan durante horas de luz y otros en horas de oscuridad. Según las horas de exposición a la luz se clasifican en plantas de día largo (altas latitudes), de día corto (bajas latitudes) o neutras, de acuerdo al tiempo diario de luz, necesario para florecer y fructificar. Varga (2018) estudió los efectos que el alumbrado ornamental puede causar sobre el algarrobo histórico de Salsacate, realizando sugerencias con el objeto de preservar el árbol y animales que lo utilizan como refugio y anidado. Las plantas son organismos dependientes de la luz que realizan diariamente un necesario “descanso fotosintético” (Baixeras, 2012).

Crecimiento. El desarrollo de las plantas está gobernado por la estacionalidad de la luz: así crecerá más rápido en primavera-verano y se estacionará en otoño-invierno con menor número de horas de luz. En invernaderos se juega con la duración diaria y el espectro de luz para incentivar crecimiento y floración. La tecnología Led significó un enorme avance por las posibilidades que brinda. Con la contaminación lumínica, las plantas están afectadas directamente, ya que los fitocromos y fotoreceptores están activados casi permanentemente, por eliminación de la alternancia luz / oscuridad.

Alteraciones de la polinización. Knop et al. (2017) consideran la luz artificial como una amenaza para la polinización. Si bien es cierto que esta disminución no es exclusiva de la iluminación artificial, el incremento de la luz nocturna y el uso 
de la iluminación Led se convirtió en una de las principales causas de la reducción de poblaciones de insectos polinizantes nocturnos. Fueron demostradas tasas de disminución superiores a 60\% en las visitas de polinizadores; a corto plazo implica disminución de producción de frutos, una influencia directa sobre la reproducción vegetal. Bariles (2019) estableció que en ambientes con mayor contaminación lumínica, la actividad polinizadora es menor.

\section{En el hombre}

Naturalmente el hombre evolucionó adaptándose a la alternacia día / noche, regularidad que comenzó a desaparecer con la invención de las lámparas y el consecuente incremento de la iluminación artificial. Este desarrollo alcanzó su máximo en el presente siglo con la masificación de la luz blanca de tecnología Led (Garcia, 2018).

Más del 80\% de la población mundial vive en condiciones de cielos contaminados por la luz artificial (Kyba, 2017). Este exceso de luz tiene influencia directa sobre la salud humana; estudios recientes demostraron que el predominante azul de la luz blanca sería responsable de obesidad, diabetes, de cierto tipo de cáncer y problemas psicológicos.

Todo tipo de iluminación artificial altera la alternancia natural de luz-oscuridad, pero particularmente el sistema led, por la predominacia de la luz azul, ya que según estudios contribuye a la reducción de formación de melatonina. Esta hormona, cuya síntesis es estimulada por la oscuridad y suprimida por la luz (Reyes-Prieto et al., 2007), actúa en la regulación del ritmo biológico o reloj interno, actividades neuroinmunológicas, oncostáticas, antiinflamatorias y antioxidantes, entre otros beneficios. En definitiva, es responsable de gran parte de nuestro estado saludable. La luz azul es un componente habitual en computadoras, teléfonos celulares, televisores con tecnología de iluminación Led. La American Medical Association ya en 2016 aconsejó utilizar las lámparas de 3000 k o menos, a fin de evitar los efectos negativos de la luz azul.

Seguridad: más iluminación no significa necesariamente más seguridad. Tenemos la idea que más iluminación es igual a más seguridad. Actualmente la tecnolo- 
gía Led permite una luminosidad superior a otras tecnologías, pero desde el punto de vista humano, no siempre contribuye a una mayor seguridad. El brillo puede disminuir la capacidad visual temporariamente (American Medical Association, 2012) y ser causa de accidentes automovilísticos. Se piensa que más iluminación es menor posibilidad de delitos, pero esto no está comprobado estadísticamente, ya que una gran parte de los delitos se produce durante el día o en la noche con muy buena iluminación. Steinbach et al. (2015) no encontraron mayores evidencias en la disminución de delitos con la utilización de iluminación led blanca de calles, comparado con el apagado total o atenuación de la misma en ciudades de Inglaterra y Gales.

Quién no sintió la incidencia de la luz de seguridad de un patrullero policial cuando debió circular detrás de uno de ellos. Resultado: fuerte molestia visual y un enceguecimiento temporario, con los consecuentes peligros para el conductor, ya que la alta intensidad de estas luminarias alargan los tiempos de acomodamiento de la visión a situaciones de menor luminosidad.

\section{Soluciones posibles}

\section{Reducción o apagado de la iluminación de carteles publicitarios.}

Desde 2013 en ciudades francesas se apagan las lucen a partir de la una de la mañana por ahorro y con la intención de disminuir el impacto ambiental (Presa \& Picicelli, 2014).

Reducción o apagado de la iluminación de calles. Tecnologías como la iluminación Led permite el atenuado de las luces en horas de menor movimiento (a partir de la medianoche) lo que reducirían efectos negativos. Incluso pueden procederse a un apagado parcial o total de la luces. En algunos países la contaminación lumínica llega al extremo de tener autopistas iluminadas en toda su extensión. La aplicación de iluminación inteligente que sólo enciende según el tránsito, sería una solución posible.

Utilización de lámparas específicas. Las lámparas led de luz azul tienen un mayor efecto negativo para los organismos. Su reemplazo por lámparas de otros colores, por ejemplo las ámbar, serán más amigables con el medio y con los orga- 
nismos. Más azul es la luz, mayor es la temperatura (fig.4); de acuerdo a numerosos estudios es el tipo de luz más perjudicial, y por lo tanto la desaconsejada y la que debe evitarse.

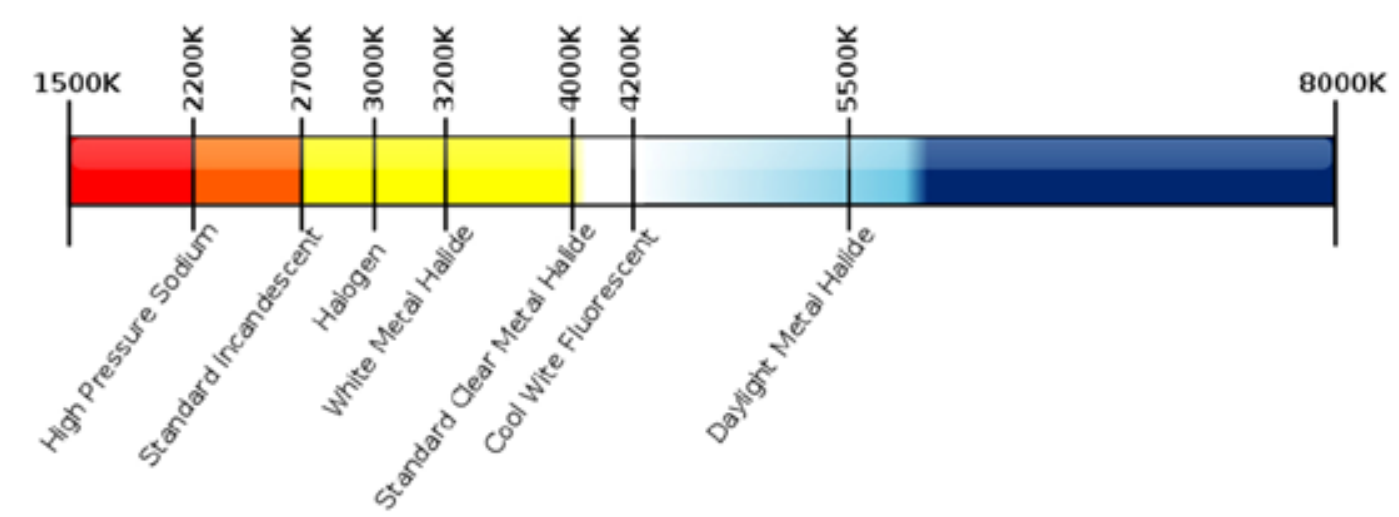

Figura 4. Carta de temperaturas kelvin de los colores . De: www.darksky.org/light-pollution/human-health/

Luminarias led de color ámbar. Las luminarias led de color ámbar permiten disfrutar del beneficio económico de esta tecnología, haciéndola más amigable con el ambiente. La luz amarillenta no se dispersa tanto como la blanca, evitando buena parte de la contaminación del cielo. Experiencias de este tipo se ven en distintas partes del mundo.

Utilización de lámparas con pantallas direccionales. Utilizar sistemas de iluminación con pantallas y un ángulo que impida la contaminación lumínica hacia el cielo nocturno, reducirá el impacto sobre aves migratorias, evitando que se desorienten.

Utilizar luces que se activen por movimiento. Los sensores de movimiento permite utilizar luces sólo cuando sea necesario, ahorrando energía y contaminando menos.

Establecimiento de normas. Presa \& Picicelli (2014) analizan la legislación argentina sobre contaminación lumínica y el desconocimiento de la población acerca de los efectos de la contaminación lumínica sobre la salud y el ambiente. Los estudios y soluciones científicas brindadas (desarrollo de nuevas lámparas más amiga- 
bles, sistemas de iluminación, intensidad, duración, periodicidad, etc.) permitirán la elaboración de normativas específicas para preservar el medio y la biodiversidad.

\section{Conclusiones}

Los efectos comprobados de la contaminación lumínica son muchos y afectan en distinto grado al ambiente, a plantas y animales, incluyendo el hombre. Los organismos han vivido con la alternancia luz / oscuridad, adaptando sus actividades y funciones. El hombre, con tecnologías de iluminación artificial, modificó esa situación natural, para lograr en poco más de un siglo el desarrollo de sistemas cada vez más eficientes, pero también cada vez más contaminantes. La contaminación lumínica y sus efectos no tuvo la atención que sí han tenido otros tipos de contaminación, probablemente por ser la menos dañina (Presa \& Picicelli, 2014). Con la irrupción masiva del sistema de iluminación Led y el incremento enorme de la superficie iluminada, se acrecentaron los problemas. Hubo así un gran interés en estudiar los efectos y tratar de solucionarlos, al menos parcialmente. Especies y ecosistemas sufrieron las consecuencias con modificaciones del reloj biológico, ritmos de descanso/actividad, reproducción, alimentación y de la salud en general.

Por otro lado, estas nuevas tecnologías contribuyen a un ahorro energético muy importante, reduciendo considerablemente la huella de carbono. Las soluciones ya están al alcance y muchas en estado de desarrollo, estableciéndose normativas referidas a aplicaciones, usos y materiales.

La aparición de sociedades científicas como la International Dark Sky Association muestran el enorme interés y la necesidad de lograr un equilibrio entre las fuentes de iluminación y el ambiente, sin dejar de lugar una fuerte concientización del uso racional de los recursos.

\section{Agradecimientos}

El proyecto PI19Foo9 Desempeño de productos de luminarias led está avalado y financiado por la Secretaría General de Ciencia y Técnica de la UNNE 


\section{Bibliografía}

Alvarez, J.E. (2018). Iluminando el futuro de la horticultura: la revolución de la tecnología LED en cultivos de interior. Noticias Secartys 31.

Aronson, S. (2020). Estudio enfoca la contaminación lumínica costera del sur de California. Sección Historia. UCLA Institute of the Environment \& Sustainability. Asociación Médica Estadounidense (2012). Informe AMA 2012.

Baixeras, A. (2012). La contaminación lumínica generada por leds blancos. Cátedra Divulgación de la Ciencia. Universitat de Valencia. P.6-8.

Bariles, J.B. (2019). Efecto de la contaminación lumínica sobre el éxito reproductivo de la especie esfingófila Caesalpinia gilliesii. Tesina. Fac.Cs.Ex., Fís,y Nat., UNC. $31 \mathrm{p}$.

Cavanillas, B. (2015). Un tipo de lámpara LED podría evitar muchas muertes en los países en desarrollo. Smartlighting, A Journal on Lighting Technologies.

Comité Español de Iluminación, p.55 a 81. Editorial MIC · www.editorialmic.com

Garcia, A. (2018). Possibles riscos de la iluminació LED per la salut humana. EspaiSalut 80. Diputació Barcelona.

Gaston, K.J, T.W.Davies, S.L.Nedelec \& L.A.Holt (2017). Impacts of artificial light at night on biological timings. Ann.Rev.of Ecology, Evolution and Systematics 48: 49-68.

Hernández Moreno (2015). Análisis comparativo por ciclo de vida de tres tipos de luminarias empleadas en los interiores de los edificios. Nova Scientia 7 (14): 538-559.

Kinver, M. (2010). Nueva York "apaga" las luces para ayudar a aves migratorias. http:// www.bbc.co.uk/mundo/ciencia_tecnologia/2010/09/100902_nueva_ york_luces_migracion_aves_jrg.shtml.

Knop, E., L. Zoller, R. Ryser, C. Gerpe, M. Hörler \& C.Fontaine (2017). Artificial light at night as a new threat to pollination. Nature 548(7666): 206-209.

Kyba, Ch.C.M., Th.Kuester, A.Sánchez de Miguel, K.Baugh, A.Jechow, F.Hölker, J. Bennie, Ch.D. Elvidge, K.J.Gaston \& L.Guanter (2017). Artificially lit surface of Earth at night increasing in radiance and extent. Science Advances 3(11): 1-9.

Lissette Ricaurte Galindo, S. (2005). ¿Existe el sueño en las aves?. Revista Electrónica de Veterinaria REDVET, Vol. 6(11): 1-13. ISSN 1695-7504. 
Malagamba Stiglich, P. (2015). Luces Led, una alternativa eficiente para optimizar la producción en invernaderos. Opinión, Agriculturers. http://agriculturers. com/luces-led-una-alternativa-eficiente-para-optimizar-la-produccion-en-invernaderos/

Pachacama Moreno, D.M. (2018). Evaluación del efecto de luces led en la propagación de dos especies de flores de verano: Gypsophila paniculata y Trachelium caeruleum. Trabajo titulación de Ing.Agropecuario. Univ. Fuerzas Armadas. Ecuador. 146 p.

Reyes-Prieto, B.M., M.Velázquez-Paniagua \& B.Prieto-Gómez (2009). Melatonina y neuropatologías. Rev. Fac. Med UNAM 52 (3): 105-109. México.

Robert, K.A., L.Partecke, J.Lesku, J.Partecke \& B.Cámaras (2015). Artificial light at night desynchronizes strictly seasonal reproduction in a wild mammal. Biología Integrativa y Comparativa. E154.

Santos, C.D., A.C.Miranda, J.P.Granadeiro, P.M.Lourenço, S.Saraiva \& J.M.Palmeirim (2010). Effects of artificial illumination on the nocturnal foraging of waders. Acta Oecologica 36(2): 166-172.

Steinbach, R., Ch.Perkins, L.Tompson, Sh.Johnson, B.Armstrong, J.Green, Ch. Grundy, P.Wilkinson \& P.Edwards (2015). The effect of reduced street lighting on road casualties and crime in England and Wales: controlled interrupted time series analysis. Journal of Epidemiology and Community Health 69 (11): 1118-1124.

Stokstad, E. (2014). LED: bueno para premios, malo para insectos. https://www. sciencemag.org/news/2014/10/leds-good-prizes-bad-insects

Ugolini , A., G.Galanti \& L.Mercatelli (2012). El gradiente de luminancia del tragaluz ayuda a los saltamontes en la identificación del sol y la luna. J. Exp. Biol. 215 (16): $2814-2819$.

Varga, T. (2018). Efectos que el alumbrado público ornamental puede causar sobre el algarrobo histórico de Salsacate. 